\title{
Efeito da aplicação de um análogo de GnRH (Buserelina) na concentração sérica de progesterona e na taxa de gestação em receptoras de embriões bovinos*
}

\section{Effect of GnRH analogue (Buserelin) on serum progesterone levels and gestation rate in bovine female receptors of embryos}

\author{
Manoel Carlos Couto de Araujo, ${ }^{\star \star}$ Eduardo Paulino da Costa, ${ }^{\star \star \star}$ Ciro Alexandre Alves Torres, ${ }^{\star \star \star \star}$ Ademir de Moraes \\ Ferreira, ${ }^{\star \star \star \star \star}$ Wanderlei Ferreira de Sá, ${ }^{\star \star \star \star \star}$ José Domingos Guimarães, ${ }^{\star \star \star}$ Francisco Aloízio Fonseca, ${ }^{\star \star \star \star}$ Deiler Sampaio \\ Costa, ${ }^{\star \star \star \star \star \star}$ Antônio Marcos Galimberti, ${ }^{\star \star}$ Célio de Freitas ${ }^{\star \star \star \star \star}$
}

\section{Resumo}

O objetivo deste trabalho foi avaliar a ação de um análogo de GnRH (Buserelina), aplicado no dia da inovulação de embriões, nos níveis plasmáticos de progesterona e, conseqüentemente, na taxa de gestação de receptoras mestiças Holandês vs Zebu. Foram realizadas cinco coletas de sangue a partir do dia da inovulação e intervaladas de 48 horas, para avaliar a concentração de progesterona. Após 50 dias da inovulação, através do exame transretal, foi observado que $60 \%$ (12/20) dos animais do grupo tratado ficaram gestantes e no grupo controle foi obtida uma taxa de gestação de $50 \%$ (10/20). Esses resultados não foram significativos pelo teste de Qui-quadrado. No entanto, os níveis séricos de progesterona dos animais do grupo tratado foram maiores $(p<0,05)$ que do grupo controle. Através dos resultados obtidos conclui-se que a aplicação de $10 \mathrm{mg}$ de buserelina no dia da inovulação do embrião causou um incremento na concentração de progesterona dos animais tratados, sem contudo resultar em aumento na taxa de gestação.

Palavras-chave: Buserelina; progesterona; taxa de gestação.

\begin{abstract}
The objective of this experiment was to evaluate the effect of GnRH analogue (buserelin), injected at the time of embryo innovulation on plasma progesterone levels and on the gestation rate of the cross bred Holandes - Zebu receptors cows. The blood sample was collected, 5 times, from the innovulation time at $48 \mathrm{~h}$ interval, in order to have a progesterone profile. The gestation rate at 50 days post-innovulation, by the transrectal examination was $50(10 / 20)$ and $60 \%(12 / 20)$ for treated and control cows which was not different among themselves. The progesterone levels were greater $(P<0.05)$ in the treated cows than in control ones. The buserelin at $10 \mathrm{mg}$ on innovulation time did affect the progesterone levels but not increase the gestation rate.
\end{abstract}

Keywords: Buserrelin; progesterone; gestation rate.

\section{Introdução}

Na pecuária brasileira, várias técnicas têm sido introduzidas visando aumentar a eficiência reprodutiva de animais geneticamente superiores, na tentativa de aumentar o número de seus descendentes e, principalmente, permitir uma expansão de oferta ao mercado consumidor. Entre estas técnicas, destaca-se a transferência de embriōes, que tem mostrado não somente ser um instrumento capaz de melhorar o de- sempenho produtivo, mas também possui grande importância no campo da investigação científica (Coelho, 1988).

Todavia, o sucesso da transferência de embriões pode ser comprometido pela mortalidade embrionária. Dentre as causas desta está a baixa concentração de progesterona $\left(P_{4}\right)$, sendo esta a maior responsável pela alta incidência de perdas embrionárias (Lukaszewska e Hansel, 1980; Lamming et al., 1989). Este esteróide gonadal é fundamental para a formação de um ambiente uterino adequado para o desen-

\footnotetext{
* Apoio financeiro da FAPEMIG - Fundação de Amparo à Pesquisa do Estado de Minas Gerais.

** Mestrando em Medicina Veterinária.

*** Departamento de Veterinária, Universidade Federal de Viçosa - CEP 36571-000 - Viçosa, MG, Brasil.

**** Departamento de Zootecnia, Universidade Federal de Viçosa.

**** Empresa Brasileira de Pesquisa Agropecuária - Gado de Leite.

\#***** Médico Veterinário (MS).
} 
volvimento do concepto (Helmer e Britt, 1986), e está relacionado também com a produção de citokinas trofoblásticas (Kerbler et al., 1997). Estas secreções trofoblásticas participam, além de processos imunológicos, também do crescimento, diferenciação e implantação do concepto (Martal et al., 1997), atuando principalmente no bloqueio da lise do corpo lúteo (Mann et al., 1995). O IFN $\tau$ é uma destas proteínas (Martal et al., 1997), o qual é primeiramente detectado entre os dias oito a dez da gestação, com sua produção chegando ao máximo em vacas entre os dias 17 e 19 (Wathes e Lamming, 1995). Outro fator produzido pelo concepto que também suprime o mecanismo de luteólise é o EDPAF (fator ativador de plaquetas derivado do embrião) (Martal et al., 1997). Conforme verificado por Battye et al. (1992) em ovelhas, esse fator impede a indução da oxitocina para a secreção de $\mathrm{PGF}_{2} \alpha$.

Com $\mathrm{o}$ intuito de evitar mortes embrionárias prematuras, a aplicação de um análogo de GnRH em receptoras de embrião poderia manter os níveis de progesterona elevados, prevenindo assim a ação luteolítica da $\mathrm{PGF}_{2} \alpha$, e proporcionando tempo para as citokinas trofoblásticas atuarem no reconhecimento materno da gestação (Drew e Peters, 1994).

A concentração de progesterona aumenta continuamente em vacas gestantes até 24 dias após a inseminação artificial, sendo que a partir do dia 10 se eleva mais em comparação com as não-gestantes (Lamming et al., 1989). Por meio da sua ação, ocorrem mudanças no ambiente uterino conducentes para o crescimento e desenvolvimento do concepto (Geisert et al.,1992). Em função disso, é provável que, se um embriāo não recebe estímulo suficiente mediado pela progesterona, teria seu desenvolvimento prejudicado, e com isso menos condições de produzir o sinal (interferons trofoblásticos) necessário para bloquear o processo luteolítico e manter a gestação (Mann et al., 1995).

Um importante problema reprodutivo em vacas e ovelhas é o alto índice de perdas embrionárias, principalmente nas três primeiras semanas de gestação (Nancarrow, 1994; Zavy, 1994). A incidência de perdas embrionárias precoces tem sido estimada em $30 \%$ a $40 \%$, com maior ocorrência antes do dia 18 (Sreenan e Diskin, 1986). Segundo Martal et al.(1997), essas perdas ocorrem quando existe uma falha na interação do concepto e o organismo materno. Assim, o desenvolvimento da gestação pode ser interrompido por um prematuro processo luteolítico causado por deficiência na percepção da presença do embrião pelo útero materno (Drew e Peters, 1994).

Um potente agonista de GnRH não-peptídico (Buserelina), com aproximadamente quarenta vezes a potência do $\mathrm{GnRH}$ endógeno (Steven, 1983) altera a função ovariana, prolongando a vida útil do corpo lúteo e a duração do ciclo estral (Milvae et al., 1984; Twagiramungo et al., 1992). Vacas tratadas com Buserelina desenvolveram um mecanismo de proteção ao corpo lúteo capaz de prevenir completamente a luteólise (Rettmer et al., 1992b). O uso desse análogo resultou em alteração da secreção das gonadotrofinas, propiciando mudanças na secreção de progesterona e estradiol (Mann e Lamming, 1995).

Objetivou-se, com este trabalho, promover um incremento nas concentrações séricas da progesterona em receptoras de embriões, via aplicação de um análogo de GNRH (buserelina) no dia da inovulação, mimetizando e proporcionando mais tempo para que o fator ativador de plaquetas derivado do embrião (EDPAF), exerça sua função luteotrófica. Objetivouse ainda aumentar, conseqüentemente, a taxa de gestação em receptoras inovuladas.

\section{Material e métodos}

O experimento foi conduzido em rebanho da EMBRAPA - Centro Nacional de Pesquisa em Bovinos de Leite, fazenda Santa Mônica, município de Vassouras, RJ. Foram utilizadas 40 fêmeas mestiças Holandês $\times$ Zebu como receptoras, com peso corporal igual ou superior a $350 \mathrm{~kg}$ e em atividade cíclica regular. As doadoras de embriões eram da raça Gir. As coletas de embriões e inovulações foram realizadas durante o segundo semestre de 1997.

As doadoras de embriões foram superovuladas com FSH-P' utilizando duas aplicações diárias a cada 12 horas em doses decrescentes, do décimo até o décimo quarto dia do ciclo estral, perfazendo um total de $\mathbf{4 0 0}$ miligramas. A aplicação do luteolítico $\left(\mathrm{PGF}_{2} \alpha\right)$ foi realizada 48 horas após o início do tratamento de superovulação. Após a manifestação do estro, foram realizadas duas inseminações, uma 12 horas após 0 início do estro e outra 48 horas após.

As coletas de embriões foram realizadas no sétimo dia após o estro, via catéter de Foley conectado em um filtro com malha de 80 micrômetros. O meio utilizado para as lavagens uterinas foi a solução de PBS de Dulbecco e Vogt, modificada por Whittinghan (1971), suplementada com $1 \%$ de soro fetal bovino. Após a coleta, com o auxílio de um microscópio estereoscópico, os embriões foram manipulados e lavados em solução de PBS modificada, suplementada com $10 \%$ de soro fetal bovino.

Após a lavagem, os embriões foram classificados quanto ao estágio de desenvolvimento segundo Lindner e Wrigth (1983) e quanto ao aspecto morfológico (Kennedy et al., 1983). Somente os embriōes de qualidade I, II e III foram utilizados para a inovulação.

As receptoras, selecionadas por meio de exames ginecológicos, foram distribuídas aleatoriamente em dọis tratamentos, contendo 20 animais cada um: Controle: Os animais receberam embriōes nos dias seis, sete e oito do ciclo estral (dia zero = dia do estro); Buserelina: As fêmeas deste grupo receberam embriōes nos dias seis, sete e oito do ciclo estral e aplicação intramuscular, no dia da inovulação, de $10 \mathrm{mg}$ de buserelina. $^{2}$

A partir do dia da inovulação foram realizadas cinco coletas de amostras de sangue com intervalos de 48 horas, utilizando tubos vacuolizados com heparina. Os tubos vacuolizados com as amostras foram acondicionados por duas a três horas em caixa de isopor contendo gelo e logo depois centrifugados a $3.000 \mathrm{rpm}$ por 15 minutos. O plasma, assim obtido, foi conservado $\mathrm{a}-10^{\circ} \mathrm{C}$ até posterior análise. As concentrações de progesterona foram dosadas por radioimunoensaio (RIA), via cintilador $\gamma$ Vitek Kineti Count 48 utilizando kit comercial ${ }^{3}$ (Coat-A Count Progesterone).

\footnotetext{
1 Pluset: Gonadorrelinas Hipofisárias Suinas - Laboratório Serono Veterinária.

${ }^{2}$ Conceptal: Buserrelina - Laboratório Hoechst Roussel Vet. S.A.

${ }^{3}$ Coat-A Count Progesterone.
} 
O diagnóstico de gestação foi realizado entre 50 e 60 dias após a inovulação, por meio de exame transretal.

Os resultados das análises de progesterona sérica de cada grupo foram analisados e comparados através de análise de variância (ANOVA), segundo Gomes (1977). A taxa de gestação verificada em cada tratamento foi analisada pelo teste do Qui-quadrado (Gomes, 1977).

\section{Resultados}

A análise da taxa de gestação pelo teste de Qui-quadrado de receptoras de embrião que foram tratadas com $10 \mathrm{mg}$ de buserelina no dia da inovulação do embrião, revelou que não houve incremento na taxa de gestação quando comparada com as receptoras que não foram tratadas (Tabela 1).

Tabela 1. Número de animais e taxa de gestação em receptoras de embriões tratadas e não-tratadas com buserelina $(10 \mathrm{~m})$ no dia da inovulação

\begin{tabular}{ccc}
\hline Grupos & $\begin{array}{c}\text { Gestantes } \\
\mathbf{n}(\%)\end{array}$ & $\begin{array}{c}\text { Nāo-Gestantes } \\
\mathbf{n}(\%)\end{array}$ \\
\hline Controle & $10(50)$ & $10(50)$ \\
Buserelina & $12(60)$ & $08(40)$ \\
\hline
\end{tabular}

Valores na mesma coluna não foram diferentes $(P>0,05)$ pelo teste de Qui-quadrado

Utilizando-se análise estatística por regressão linear múltipla, constatou-se que houve um aumento na concentração plasmática de progesterona $(P<0,05)$ nos animais tratados com buserelina, quando comparados com os animais do grupo controle, a qual é melhor explicada por meio de uma equação do tipo linear (Figura 1).

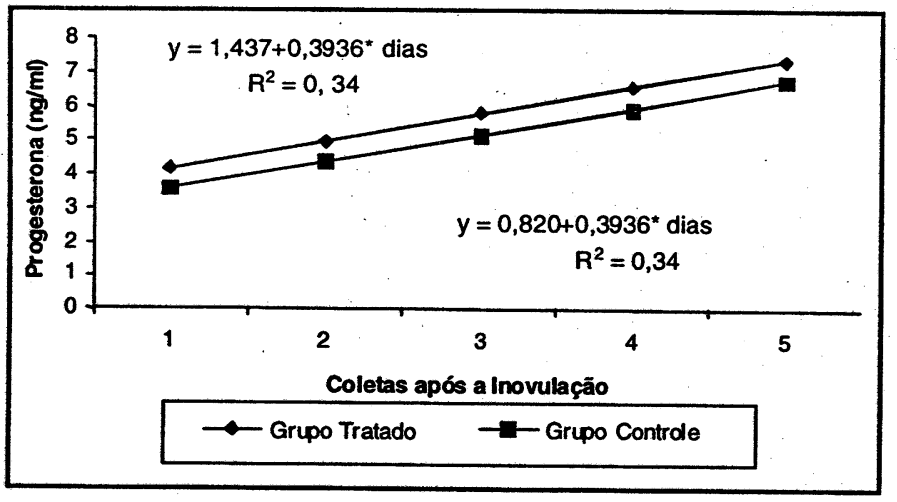

Figura1: Concentração de progesterona, a partir da inovulação, em receptoras submetidas ou não à aplicação de $10 \mathrm{mg}$ de buserelina no dia da inovulação.

\section{Discussão}

Segundo Mann e Lamming (1995), o aumento nos níveis de $\mathrm{P}_{4}$ pode ser um fator importante para aumentar a taxa de gestaçăo, pois maiores quantidades desse esteróide poderiam exercer uma função luteoprotetora e prevenir a regressão luteal (Rettmer et al., 1992a). Corroborando com estes autores,
Peters (1996) afirma que a manutenção da secreção de progesterona por um corpo lúteo viável é imprescindível no início da gestação e, provavelmente, a luteólise prematura é uma das causas principais de morte embrionária.

Apesar de promover uma elevação nos níveis de $P_{4}(P<0,05)$, a aplicação de $10 \mu \mathrm{g}$ de buserelina, em receptoras, no dia da transferência de embriões, não causou aumento na taxa de gestação dos animais tratados em comparação com os animais do grupo controle. Os resultados deste experimento são semelhantes aos encontrados por Macmillan et al. (1986) quando aplicaram $10 \mu \mathrm{g}$ de buserelina entre $07^{\circ} \mathrm{e} o$ $10^{\circ}$ dia do ciclo estral após a inseminação artificial, e aos de Drew e Peters (1994), realizando o tratamento no $8^{\circ}$ e $10^{\circ}$ dia após a I.A.

A falha em aumentar a taxa de gestação após a aplicação de buserelina, no dia da inovulação, possivelmente esteja relacionada com o dia do ciclo, pois quando a aplicação desse GnRH agonista foi realizada entre o $11^{\circ}$ e $13^{\circ}$ dias após I.A.; observou-se elevação na taxa de gestação (Macmillan et al., 1986), o que também ocorreu quando foi realizado o tratamento de $10 \mu \mathrm{g}$ de buserelina somente no $11^{\circ}$ dia (Sheldon e Dobson, 1993) ou no $12^{\circ}$ dia posterior à I.A (Drew e Peters, 1994).

A importância do dia da aplicação poderia ser explicada em função da duração do efeito antiluteolítico da progesterona, que tem sido relatada ser de oito a 10 dias (Wathes $e$ Lamming, 1995), embora Peters (1996) tenha observado um período de 10 a 12 dias. Segundo Wathes e Lamming (1995), a progesterona estimula a formação de inibidores endometriais neste período, os quais bloqueiam a formação de receptores locais para a oxitocina (Peters, 1996). A formação destes receptores é o início da luteólise, pois, após a sua formação, ocorre aumento no padrão pulsátil de $\mathrm{PGF}_{2} \alpha$ (Lamming e Mann 1993). Em função disto, Peters (1996) afirma que o tratamento com buserelina teria efeito sobre a fertilidade apenas quando realizado entre o $11^{\circ}$ e $13^{\circ} \mathrm{dia}$, pois iria inibir as pulsações de $\mathrm{PGF}_{2} \alpha$, impedindo a luteólise e beneficiando a ação dos interferons secretados pelo embrião, responsáveis pelo reconhecimento materno da gestação. Estas observações estão de acordo com as de Harvey et al. (1994), os quais relataram que a aplicação deste análogo neste período produz elevadas concentrações de progesterona na fase crítica de reconhecimento materno da gestação. Apesar de alguns pesquisadores não terem encontrado incremento nos níveis séricos de progesterona em animais submetidos a uma aplicação de $10 \mu \mathrm{g}$ de buserelina entre os dias 11 e 13 do ciclo (Mann e Lamming, 1995), o uso de outro análogo do GNRH $(200 \mu \mathrm{g}$ de acetato de fertirelina) neste mesmo período promove aumento na concentração de progesterona quatro a 12 dias após a injeção, com maior concentração e pico nos animais tratados (Rettmer et al., 1992a). Adicionalmente, Rettmer et al. (1992b) observaram aumento na taxa de gestação com a aplicação de 100 ou $200 \mu \mathrm{g}$ de fertirelina em uma única dose, nos dias 11 a 14 do ciclo. Entretanto, estes pesquisadores verificaram maior taxa quando a aplicação era realizada nos dias 12 ou 13, ao invés dos dias 11 ou 14. Corrobora com estes achados a ausência de incremento na taxa de gestação de animais inseminados, após a aplicação de buserelina entre os dias sete e 10 do ciclo (Macmillan et al., 1986; Drew e Peters, 1991). Baseando-se nas pesquisas 
relatadas, é provável que o dia utilizado para a aplicação de buserelina, no presente experimento, não tenha tido efeito bloqueador na formação de receptores de oxitocina de forma necessária, ou por tempo suficiente, para inibir a luteólise e beneficiar os fatores do reconhecimento materno.

Além desta possibilidade, Drew e Peters (1994) declaram que os padrões relativos ao desenvolvimento folicular também podem determinar as diferenças nos grupos que respondem e nos que não respondem ao tratamento com um $\mathrm{GnRH}$ agonista. Segundo esses pesquisadores, o tratamento no dia 12, em animais que apresentam três ondas de crescimento folicular, teria ocorrido quando o tamanho do folículo da segunda onda estaria, no máximo, prejudicando a produção de estradiol e, conseqüentemente, inibindo a ação de $\mathrm{PGF}_{2} \alpha$ que começa ser secretada, pois o estradiol estimula a formação de receptores para oxitocina no endométrio uterino (Drew e Peters, 1994). Sendo assim, a diminuição na concentração sérica de estradiol seria um fator importante para melhorar a taxa de gestação (Harvey et al., 1994). Mann e Lamming (1995) concluem que o tratamento com buserelina aumenta as chances de sobrevivência do embrião e melhora as taxas de gestação por causar uma diminuição na concentração de estradiol, reduzindo o estímulo para o desenvolvimento do mecanismo luteolítico. Dessa forma, é provável que a aplicação de buserelina no dia da inovulação seja uma prática prematura pois, possivelmente, não causaria uma redução nas concentrações de estradiol de forma a inibir a produção de $\mathrm{PGF}_{2} \alpha$ e bloquear o processo luteolítico, o qual

\section{Referências bibliográficas}

BATTYE, K.M., O'NEILL, C., EVANS, G. Evidence that platelet-activating factor supresses uterine oxytocin-induced 13, 14-dihydro15ketoprosta-glandin $\mathrm{F}_{2}$ a release and phosphatidylinositol hydrolysis in the ewe. Biology of Reproductionv. 47, p. 213-219, 1992.

COELHO, S.G. Transferência de embriōes de raças zebuínas. Dissertação (mestrado) - Belo Horizonte, Escola de Veterinária da UFMG, 1988.

DREW, S.B., PETERS, A.R. Effect of treatment with a gonadotrophin releasing hormone analogue on the fertility of dairy cows. British Society of Animal Production, p. 1121-1123, 1991.

DREW, S.B., PETERS, A.R. Effect of buserelin on pregnancy rates in dairy cows. Veterinary Record. v. 134 p. 267-269, 1994.

GEISERT, R.D., MORGAN, G.L., SHORT, E.C., ZAVY, M.T. Endocrine events associated with endometrial function and conceptus development in cattle. Reprod. Fertil. Dev. v. 4, p. 301-305, 1992.

GOMES, F. P. Curso de estatística experimental. 7. ed. Piracicaba: Nobel, $1977.430 \mathrm{p}$.

HARVEY, M.J., RENTON, J.P., SALAHEDDINE, M., ROBERTSON, L. Ovarian and clinical response of cattle to buserelin. The Veterinary Record. v. 134, p. 168-171, 1994.

HELMER, S.D., BRITT, J.H. Fertility of dairy cattle treated with human chorionic gonadotrophin ( $\mathrm{hCG}$ ) to stimulate progesterone secretion. Theriogenology, v. 26, p. 683-695, 1986.

JUBB, T.F., ABHAYARATNE, D., MALMO, J., ANDERSON, G.A. Failure of intramuscular injection of an analogue of gonadotrophin-releasing hormone 11 to 13 days after insemination to increase pregnancy rates in dairy cattle. Australian Veterinary Journal. v. 67, p. 359$361,1990$. se inicia a partir do dia 12 do ciclo estral (Drew e Peters, 1994).

Destarte, a aplicação de buserelina no dia da inovulação não prolongou a atividade luteal pelo seu efeito luteotrófico e antiluteolítico, de forma a contribuir para que os fatores do reconhecimento materno, secretados a partir do $12^{\circ}$ e $13^{\circ}$ dia do ciclo (Peters, 1996), tivessem melhores condições de atuar, aumentando a taxa de gestação.

Entretanto, outros fatores, além do dia da aplicação da buserelina, também poderiam interferir no efeito do tratamento com este agonista do GnRH. Assim, Jubb et al. (1990), realizando o experimento similar ao de Rettmer et al. (1992b), não encontraram elevação na taxa de gestação. Um aspecto a ser considerado é que embriões morfologicamente viáveis podem ter alterações não-visiveis, as quais poderiam interferir na capacidade de sobrevivência após a transferência. Adicionalmente, a precisão na identificação do estro e as diferenças entre os rebanhos tratados também poderiam interferir no efeito do tratamento com análogos do GnRH (Rettmer et al. 1992b; Drew e Peters, 1994).

\section{Conclusões}

A aplicação de $10 \mu \mathrm{g}$ de buserelina em receptoras de embriões no dia da inovulação causa um aumento significativo nas concentrações plasmáticas de progesterona. No entanto, o tratamento com esse análogo do $\mathrm{GnRH}$ no dia referido não promove incremento na taxa de gestação.

KENNEDY, C. G., BOLAND, M. P., GORDON, I. The effect of embryo quality of freezing on subsequent development of thawed cow embryo. Theriogenology, v. 19, p. 823-832, 1983.

KERBLER, T.L., BUHR, M.M., JORDAN, L.T., LESLIE, K.E., WALTON, J.S. Relationship between maternal plasma progesterone concentration and interferon-tau synthesis by the conceptus in cattle. Theriogenologyv. 47, p. 703-714, 1997.

LAMMING, G. E., DARWASH, A.O., BACK, H.L. Corpus luteum function in dairy cows and embryo mortality. J Reprod. Fertil. Suppl. v. 37, p. 245-252, 1989.

LAMMING, G.E., MANN, G.E. Progesterone concentration affects the development of the luteolytic mechanism in the cow. J. Reprod. Fertil. Abstr. Ser. v. 11, p. 8, 1993.

LINDNER, G. M., WRIGHT Jr, R. W. Bovine embryo morphology and evaluation. Theriogenology., v. 20, n. 4, p. 407-416, 1983.

LUKASZEWSKA, J., HANSEL, W. Corpus luteum maintenance during early pregnancy in the cow: J. Reprod. Fertil. v. 59, p. 485-493, 1980.

MACMILLAN, K.L., TAUFA, V.K., DAY, A.M. Effects of an agonist of gonadortropin releasing hormone (buserelin) in cattle. III. Pregnancy rates after rates a post-insemination injection during metaestrous or dioestrus. Animal Reproduction Science. v.11, p. 1-10, 1986.

MANN, G.E. \& LAMMING, G.E. Effects of treatment with buserelin on plasma concentrations of oestradiol and progesterone and cycle length in the cow. British Veterinary Journal. v. 151 p. 427-432, 1995.

MANN, G.E., LAMMING, G.E., FRAY, M.D. Plasma oestradiol and progesterone during early pregnancy in the cow and the effects of treatment with buserelin. Animal Reproduction Science. v. 37, p. 121-131, 1995 
MARTAL, J., NICOLE, C., SYLVAINE, C., HUYNH, L., LANTIER, F., HERMIER, P., HARIDON, R.L., CHARPIGNY, G., CHARLIER, M., CHAOUAT, G. Recent developments and potentialities for reducing embryo mortality in ruminants: the role of IFNt and other citokines in early pregnancy. Reprod. Fertil. Dev. v. 9, p. 355-380, 1997.

MILVAE, R. A.; MURPHY, B. D.; HANSEL, W. Prolongation of bovine estrous cycle with a gonadotropin releasing hormone analog. Biol. Reprod., v. 31, p. 664-670, 1984.

NANCARROW, C.D. Embryonic mortality in the ewe and doe. In: ZAVY, M.T., GEISERT, R.D. (eds), Embryonic Mortality in Domestic Species. Boca Raton, FL: CRC Press. p. 79-97, 1994.

PETERS, A.R. Embryo mortality in the cow. AnimalBreeding Abstracts, v. 64, n. 8, p. 587-597, 1996.

RETTMER, I., STEVENSON, J.S., CORAH, L.R. Pregnancy rates in beef cattle after administering a GnRH agonist 11 to 14 days after insemination. J. Anim. Sci. v. 70, p. 7, 1992a.

RETTMER, I., STEVENSON, S., LARRY, R.C. Endocrine responses and ovarian changes in inseminated dairy heifers after an injection of a GnRH agonist 11 to 13 days after estrus. J. Anim. Sci. v. 70, p. 508517, $1992 b$.
SHELDON, I.M., DOBSON, $H$. Effects of gonadotrophin releasing hormone administered 11 diays after insemination on the pregnancy rates of cattle to the first and later sevices Veterinary Record, $\mathrm{v}$. 14, p. 160-163, 1993.

SREENAN, J.M., DISKIN, M.G. Factors affecting pregnancy rate following embryo transfer in the cow. Theriogenology, v. 27, n. 1, p. 99-113, 1987.

STEVEN J. O. Clinical uses of luteinizing hormone - releasing hormone. Fertility \& Sterility, v. 39, n. 5, p. 577-590, 1983.

TWAGIRAMUNGO, H., GUILBAULT, L. A., PROULX, J., DUFOUR, J. J. Synchronization of estrus and fertility in beef cattle with two injections of buserelin and prostaglandin. Theriogenology, v. 38, p. 1131-1144, 1992.

WATHES, D.C., LAMMING, G.E. The oxytocin receptor, luteolysis and the maintenance of pregnancy. Journals of Reproduction and Fertilty, v. 49, p. 53-57, 1995.

WHITTINGHAM, D. G. Survival of mouse embryos after freezing and thawing. Nature, v. 233, p. 125, 1971.

ZAVY, M.T. Embryonic mortality in cattle. In: ZAVY, M.T., GEISERT, R.D. (Eds.), Embryonic Mortality in Domestic Species. Boca Raton, FL: CRC Press. p. 99-140, 1994. 